\title{
Variação espacial e temporal na diversidade e composição de espécies de peixes em riachos da bacia do Rio Ivinhema, Alto Rio Paraná
}

\author{
Yzel Rondon Súarez ${ }^{1,2}$ \\ ${ }^{1}$ Laboratório de Ecologia, Centro Integrado de Análise e Monitoramento Ambiental - CINAM \\ Universidade Estadual de Mato Grosso do Sul - UEMS, \\ Rod. Dourados-Itahum, Km 12, CEP 79804-970, Dourados, MS, Brasil \\ ${ }^{2}$ Autor para correspondência: Yzel Rondon Súarez,e-mail: yzel@uems.br
}

SÚAREZ, Y.R. 2008. Spatial and temporal variation in fish species diversity and composition in streams of Ivinhema River basin, upper Paraná River. Biota Neotrop. 8(3): http://www.biotaneotropica.org.br/v8n3/ en/abstract?article+bn02308032008.

\begin{abstract}
Aiming to describe the spatial and temporal patterns of the fish species diversity and composition in seven streams of Ivinhema basin, upper Paraná River, monthly samples were realized from January to December/2002. Forty six fish species were found, being estimated the occurrence of fifty five species in these streams. The species richness and evenness did not present seasonal variation, however, presented spatial (between streams) variations. The Analysis of Similarity (ANOSIM) also suggested that only spatial variation in species composition is significant. The species richness presented significant correlation with the stream width $(\mathrm{r}=0.80)$ and depth $(\mathrm{r}=0.78)$. The evenness presented significant correlation only in mean number of collected individuals $(\mathrm{r}=-0.91)$. Only stream width explained significantly the distribution of main fish species in the sampled streams. Some species as $H$. marginatus, A. dentatus, $M$. sanctaefilomenae occurred mainly in larger streams and A. fasciatus and $B$. stramineus occurred mainly in shallow streams and with high water velocity, indicating the importance of hydrological characteristics in species distribution. The physical characteristics of the streams are more important than temporal variations in fish assemblages organization of sampled streams.
\end{abstract}

Keywords: neotropical fish, species diversity, beta diversity, communities structure.

SÚAREZ, Y.R. 2008. Variação espacial e temporal na diversidade e composição de espécies de peixes em riachos da bacia do Rio Ivinhema, Alto Rio Paraná. Biota Neotrop. 8(3): http://www.biotaneotropica.org.br/ v8n3/pt/abstract?article+bn02308032008.

Resumo: Com o objetivo de descrever o padrão espacial e temporal na diversidade e composição de espécies de peixes em sete riachos da bacia do rio Ivinhema, alto rio Paraná, amostragens mensais foram realizadas entre janeiro e dezembro/2002. Quarenta e seis espécies de peixes foram encontradas, sendo estimada a ocorrência de cinquienta e cinco espécies nestes riachos. A riqueza de espécies e a equitabilidade não apresentaram variações temporais significativas, contudo, foi constatada variação espacial (entre riachos) significativa. A Análise de Similaridade (ANOSIM) sugere que existe somente variação espacial significativa na composição de espécies. A riqueza de espécies foi significativamente correlacionada com a largura $(\mathrm{r}=0,80)$ e a profundidade do riacho $(\mathrm{r}=0,78)$. A equitabilidade apresentou correlação significativa somente com o número médio de indivíduos coletados $(\mathrm{r}=-0,91)$. Apenas a largura dos riachos influenciou significativamente a distribuição das principais espécies de peixes nos riachos amostrados. Algumas espécies como H. marginatus, A. dentatus, M. sanctaefilomenae ocorreram principalmente em riachos mais volumosos e $A$. fasciatus e $B$. stramineus ocorreram principalmente em riachos rasos e com elevada velocidade da correnteza, indicando a importância das características hidrológicas na distribuição das espécies. As características físicas dos riachos são mais importantes que as variações temporais na organização das assembléias de peixes dos riachos amostrados.

Palavras-chave: peixes neotropicais, diversidade de espécies, diversidade beta, estrutura de comunidades. 


\section{Introdução}

Estudos recentes sobre ecologia de comunidades têm enfatizado que as comunidades locais são determinadas por processos que atuam em múltiplas escalas espaciais e temporais (Heino et al. 2007). Desta forma, dependendo do enfoque e da escala analisada várias características têm sido citadas como determinantes da estrutura das comunidades de peixes em riachos, desde características de estrutura dos habitats (Gorman \& Karr 1978, Angermeier \& Karr 1983), interações bióticas (Zaret \& Rand 1971, Rahel \& Stein 1988) ou filtros ambientais (Poff 1997).

A despeito do crescimento do número de trabalhos quantificando a importância de fatores bióticos, abióticos e biogeográficos sobre as comunidades de peixes em regiões temperadas (Rahel \& Stein 1988, Matthews \& Robison 1998, Taylor \& Warren 2001, Oberdorff et al. 2001), poucos estudos têm sido realizados em comunidades neotropicais. Desta forma, a quantificação da importância das variações espaciais e temporais sobre as comunidades aquáticas é de grande importância para a compreensão dos mecanismos estruturadores dessas comunidades e para fornecer subsídios para prever o impacto da influência antrópica sobre esses ecossistemas.

A bacia do alto rio Paraná é considerada uma área de endemismo para vários grupos de peixes, devido à formidável barreira migratória representada, até recentemente, pela cachoeira de Sete Quedas, que isolaram por muito tempo a maioria da ictiofauna do alto rio Paraná da fauna remanescente dos sistemas dos rios La Plata-UruguaiParaná-Paraguai (Britski \& Langeani 1988, Weitzman et al. 1988, Castro \& Casatti 1997). Este endemismo, associado à dependência da vegetação ripária, torna estas comunidades altamente vulneráveis aos impactos ambientais decorrentes de ações antrópicas, principalmente para fins de produção de energia elétrica e agropecuária, sendo que vários estudos têm encontrado alterações na estrutura das comunidades de peixes em função das alterações antrópicas (Casatti 2004, Wright \& Flecker 2004, Cetra \& Petrere Júnior 2006, Ferreira \& Casatti 2006, Casatti et al. 2006), sem que a dinâmica de variação na diversidade e composição de espécies seja devidamente conhecida, o que dificulta a elaboração de programas de uso sustentável dos recursos aquáticos.

No trecho entre a hidrelétrica de Porto Primavera e a de Itaipu (único trecho do alto rio Paraná livre de grandes represamentos), poucos estudos sobre as comunidades de peixes de riachos foram realizados, destacando-se os trabalhos de Penckzak et al. (1994), Agostinho \& Penczak (1995), Abes \& Agostinho (2001) e Pavanelli \& Caramaschi (2003) em riachos da planície de inundação do rio Paraná; Súarez \& Petrere (2005) em riachos da bacia do rio Iguatemi; Valério et al. (2007) e Súarez et al. (2007) em riachos de cabeceira da bacia do alto rio Paraná (bacia do rio Ivinhema) no Mato Grosso do Sul, assim como Súarez (2008) que lista as espécies de peixes presentes nos riachos analisados no presente estudo.

Assim, considerando o pouco conhecimento das comunidades de peixes da bacia do rio Ivinhema, alto rio Paraná, o presente trabalho teve como objetivo responder às seguintes questões: 1) Existe variação temporal e espacial significativa na composição e diversidade de espécies de peixes nestes riachos? Qual destas fontes de variação é a mais importante? 2) Como as características dos riachos influenciam a diversidade e composição de espécies de peixes nos riachos da porção inferior da bacia do rio Ivinhema?

\section{Material e Métodos}

As amostragens foram realizadas mensalmente em sete locais em duas micro-bacias da bacia do rio Ivinhema, alto rio Paraná (Figura 1) entre janeiro e dezembro de 2002. As amostragens foram realizadas com tela de isca $(1,2 \times 0,8 \mathrm{~m})$, que é uma peneira retangular de armação metálica e tela mosquiteiro ( $2 \mathrm{~mm}$ de abertura de malha) sendo realizados 20 lances por local. Cada lance consistia em passar a tela rente ao fundo do riacho no sentido meio-margem, forçando a tela contra as margens desalojando os peixes escondidos sob as plantas aquáticas e gramíneas nas margens. Todos os riachos foram amostrados no período diurno em um trecho de aproximadamente $50 \mathrm{~m}$. No mês de janeiro não foi realizada amostragem no Córrego da Mata, devido a problemas logísticos. As principais características dos riachos amostrados são apresentadas na Tabela 1.

Os peixes capturados foram fixados em formol a $10 \%$ e posteriormente transferidos para álcool a $70^{\circ} \mathrm{GL}$. As identificações foram realizadas com auxílio dos trabalhos de Britski et al. (1999) e Graça e Pavanelli (2007), além de chaves específicas para cada grupo taxonômico e comparações com espécimes testemunho no Museu de Zoologia da Universidade de São Paulo (MZUSP), onde exemplares testemunho de algumas espécies foram depositados.

A fim de quantificar a importância da variação espacial e temporal sobre a riqueza de espécies (aqui definida como número de espécies), foram realizadas Análises de Co-Variância (ANCOVA) tendo como variáveis explanatórias (categorias) os riachos e os meses amostrados e como co-variável o número de indivíduos coletados. Devido à ausência de normalidade, a variação espacial e temporal na equitabilidade foi quantificada através de testes de Kruskall-Wallis. A equitabilidade foi definida como H'/Hmax, onde H'é o índice de diversidade de Shannon e Hmax (diversidade máxima) é o logaritmo da riqueza de espécies, em ambas as estimativas de diversidade foram utilizados os valores de $\log 2$.

Para verificar se existe diferença espacial e temporal significativa na composição de espécies nos riachos estudados, foi realizada uma Análise de Similaridade (ANOSIM), proposta por Clarke (1993).

A quantificação da influência das características ambientais (largura, profundidade, velocidade e transparência da água) bem como do número médio de indivíduos coletados sobre a diversidade de espécies (riqueza total, equitabilidade média e diversidade $\beta$ ) foi quantificada através de uma análise de correlação de Pearson. A diversidade $\beta$ foi definida como: riqueza total menos riqueza média $(\beta=\gamma-\alpha)$, de acordo com a metodologia proposta por MacArthur et al. (1966) e neste caso passa a assumir uma medida de "turnover" na comunidade. Desta forma, locais que apresentam elevada riqueza total (ano todo), mas baixo número médio de espécies por coleta devem apresentar alta taxa de alteração temporal na composição de espécies (elevada diversidade $\beta$ ).

Os dados de abundância total das espécies (soma das 12 amostragens) nos riachos foram utilizados em uma análise de agrupamento (Coeficiente de Bray Curtis), através do método de ligação UPGMA, a fim de evidenciar os padrões de similaridade na composição de espécies entre os riachos. O método de ligação UPGMA foi escolhido por minimizar a distorção da matriz inicial de similaridade na construção do dendrograma enquanto o coeficiente de Bray-Curtis foi utilizado por ser considerado o mais eficiente na realização da ANOSIM (Clarke 1993) e desta forma a ANOSIM e a representação gráfica da similaridade na composição de espécies entre os riachos deveriam ser apresentadas utilizando o mesmo coeficiente.

A influência da autocorrelação espacial sobre a composição de espécies de peixes foi analisada através de um teste de Mantel (999 permutações) quantificando a influência da distância entre os locais amostrados sobre a similaridade na composição de espécies entre os riachos.

A influência das características dos riachos amostrados sobre a abundância das principais espécies de peixes (pelo menos $1 \%$ do total de indivíduos coletados) foi quantificada através de uma análise de correspondência canônica (CCA). As análises foram realizadas utilizando o software R (R Development Core Team 2005). Para 


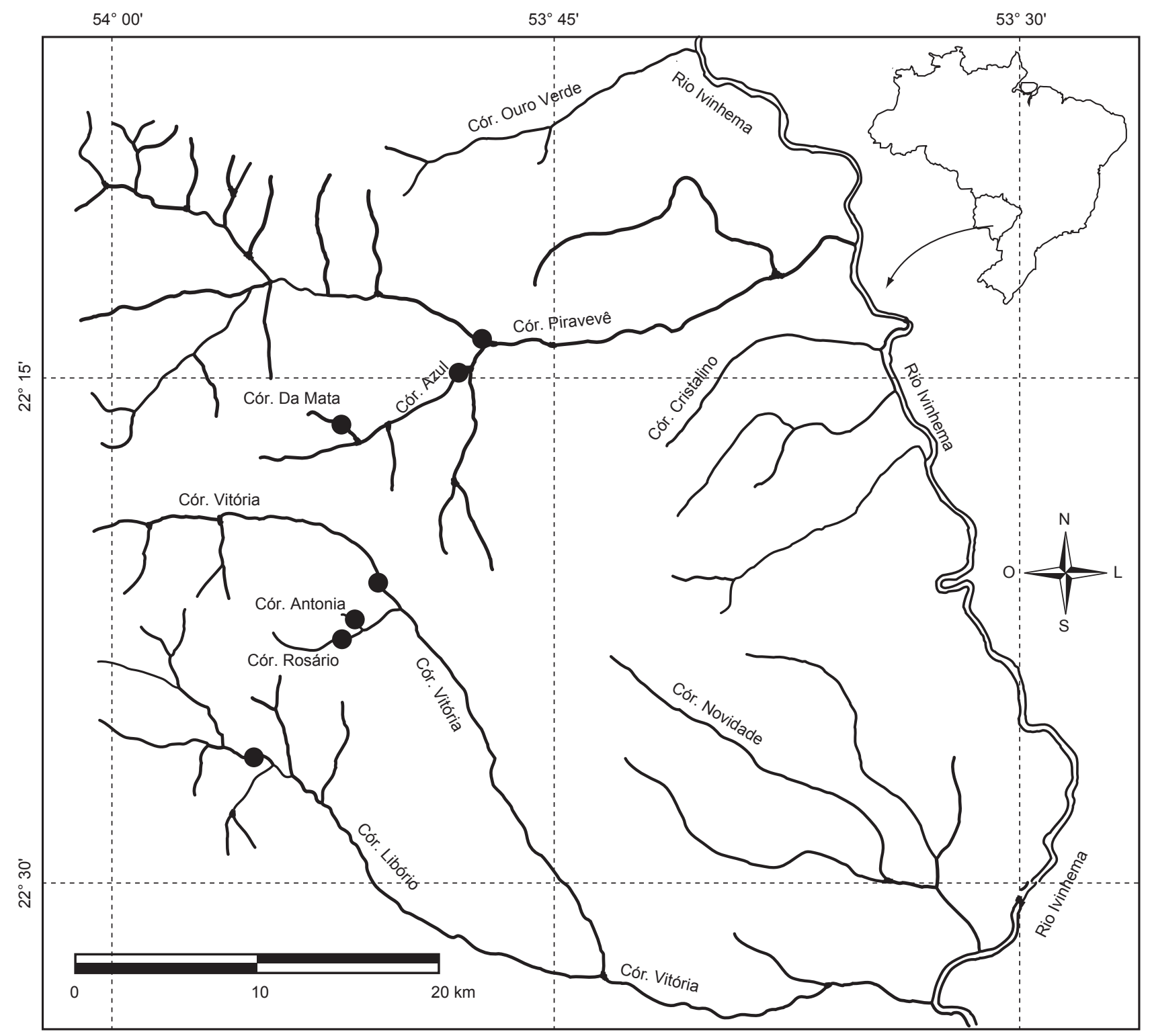

Figura 1. Localização dos riachos amostrados na bacia do rio Ivinhema, alto rio Paraná, Mato Grosso do Sul.

Figure 1. Locations of the sampled streams in the Ivinhema River basin, upper Paraná River, Mato Grosso do Sul.

definir a significância da influência das variáveis ambientais sobre a distribuição em abundância das espécies foi quantificada através da rotina "envfit" no software "vegan" que após a obtenção de um valor de $r^{2}$ para as variáveis ambientais utiliza um processo de randomização para definir a significância destas variáveis (999 permutações) para todos os eixos em conjunto.

\section{Resultados}

Foram coletados 3.383 indivíduos, distribuídos em 46 espécies. Hemigrammus marginatus Ellis, 1911 foi a espécie mais abundante com 956 indivíduos (28,3\%), seguida de Serrapinnus notomelas (Eigenmann, 1915) com 476 indivíduos (14,1\%). Enquanto cinco espécies representaram $67,7 \%$ dos indivíduos coletados outras nove espécies apresentaram apenas um indivíduo em todo o período amostrado.
A riqueza total nos riachos estudados foi significativamente correlacionada com a largura $(r=0,80)$ e profundidade $(r=0,78)$ dos riachos. A diversidade $\beta$ foi correlacionada com a largura $(r=0,90)$ e profundidade $(r=0,79)$ dos riachos enquanto a equitabilidade média foi negativamente correlacionada com o número médio de indivíduos coletados $(r=-0,91)$ (Tabela 2). Desta forma, riachos com maior profundidade e largura apresentam maior riqueza de espécies e maior diversidade beta, enquanto riachos com maior número de indivíduos apresentam menor equitabilidade (Figura 2).

Não constatamos variação temporal significativa nos parâmetros analisados das comunidades, contudo a variação espacial foi significativa tanto para a riqueza de espécies quanto para a equitabilidade (Tabela 3 e Figura 3).

Constatamos através da Análise de Similaridade que não existe diferença temporal significativa na composição de espécies $(\mathrm{R}=-0,048 ; \mathrm{P}=0,943)$, no entanto, existe diferença significativa entre os riachos estudados $(R=0,663 ; P=0,001)$, sendo que o 
Tabela 1. Características gerais dos riachos amostrados na bacia do rio Ivinhema, Alto Rio Paraná: ordem do canal (conforme convenção de Strahler), velocidade da corrente $(\mathrm{m} / \mathrm{s})$, transparência média da água $(\mathrm{m})$, profundidade média $(\mathrm{m})$ e largura média $(\mathrm{m})$.

Table 1. General characteristics of sampled streams in the Ivinhema River Basin, Upper Paraná Basin. Stream order (according Strahler), water velocity (m/s), mean water transparency $(\mathrm{m})$, mean depth (m) and mean width (m).

\begin{tabular}{|c|c|c|c|c|c|c|}
\hline Riachos & Ordem & Veloc. & Transp. & Prof. & Larg. & Vegetação \\
\hline Cór. Libório & 2 & 0,30 & 0,23 & 0,75 & 4,5 & $\begin{array}{l}\text { Mata à montante, poucas gramíneas nas } \\
\text { margens }\end{array}$ \\
\hline Cór. Antonia & 1 & 0,40 & 1,20 & 0,50 & 1,2 & Mata fechada, sem macrófitas aquáticas \\
\hline Cór. Rosário & 1 & 0,50 & 1,40 & 1,40 & 2,0 & $\begin{array}{l}\text { Sem mata, com grandes bancos de gramíneas } \\
\text { flutuantes e Typha sp. em trecho onde o córrego } \\
\text { forma área alagada }\end{array}$ \\
\hline Cór. Vitória & 2 & 0,10 & 0,35 & 0,80 & 3,8 & $\begin{array}{l}\text { Pouca mata, com muitas gramíneas nas } \\
\text { margens }\end{array}$ \\
\hline Cór. Piravevê & 3 & 0,10 & 0,25 & 2,50 & 8,0 & $\begin{array}{l}\text { Pouca mata, com muitas gramíneas nas margens, } \\
\text { pequena lagoa marginal e área alagável }\end{array}$ \\
\hline Cór. Azul & 2 & 0,35 & 0,33 & 0,78 & 3,0 & $\begin{array}{l}\text { Sem mata, com muitas gramíneas e Eichhornia } \\
\text { azurea na margem }\end{array}$ \\
\hline Cór. Da Mata & 1 & 0,20 & 0,60 & 0,50 & 1,5 & $\begin{array}{l}\text { Curso alterado por construção de açude e } \\
\text { desmatamento, muitas Gramíneas nas margens }\end{array}$ \\
\hline
\end{tabular}

Tabela 2. Correlação de Pearson entre os parâmetros das comunidades e as características dos riachos estudados e número de indivíduos na bacia do rio Ivinhema, Alto Rio Paraná, entre Janeiro e Dezembro/2002.

Table 2. Pearson correlation between communities descriptors and streams characteristics in Ivinhema river basin and number of individuals, Upper Paraná Basin, from January to December/2002.

\begin{tabular}{lccc}
\hline \multicolumn{1}{c}{ Variáveis } & Riqueza & Diversidade $\beta$ & Equitabilidade \\
\hline Indivíduos & $0,70 \mathrm{~ns}$ & 0,61 & $-0,91^{* *}$ \\
Velocidade & $-0,44 \mathrm{~ns}$ & $-0,54$ & $0,53 \mathrm{~ns}$ \\
Transparência & $-0,55 \mathrm{~ns}$ & $-0,65$ & $0,08 \mathrm{~ns}$ \\
Profundidade & $0,78^{*}$ & $0,79 *$ & $-0,75 \mathrm{~ns}$ \\
Largura & $0,80^{*}$ & $0,90^{* *}$ & $-0,50 \mathrm{~ns}$ \\
\hline
\end{tabular}

ns = Não significativo; $*$ = Significativo a alfa 0,$05 ; * *=$ Significativo a alfa 0,01 .

$\mathrm{ns}=$ Non significant $; *=$ Significantly to alfa $0.05 ; * *$ Significantly to alfa 0.01 .

dendrograma de similaridade entre os riachos, indica diferenciação entre as microbacias estudadas, desta forma, com exceção do córrego Rosário, os demais locais se diferenciaram pela micro-bacia hidrográfica (Figura 4). Contudo, apesar da diferenciação entre microbacias observada no dendrograma de similaridade, não constatamos influência da distância entre os locais sobre a composição de espécies (Mantel $\mathrm{r}=0,31 ; \mathrm{P}=0,09$ ).

Por meio da análise de correspondência canônica foi obtido que $59,8 \%$ da variação na abundância das principais espécies de peixes podem ser explicadas pelas características dos riachos estudados, sendo que o primeiro eixo explicou $39 \%$ da variação nos dados enquanto o segundo eixo explicou $20,8 \%$ da variação nos dados. Contudo, dos quatro descritores dos riachos utilizados apenas a largura dos riachos influenciou significativamente a composição de espécies (Tabela 4).

Entre as espécies analisadas, Serrapinnus notomelas, Hisonotus sp., Aphyocharax dentatus Eigenmann \& Kennedy, 1903,
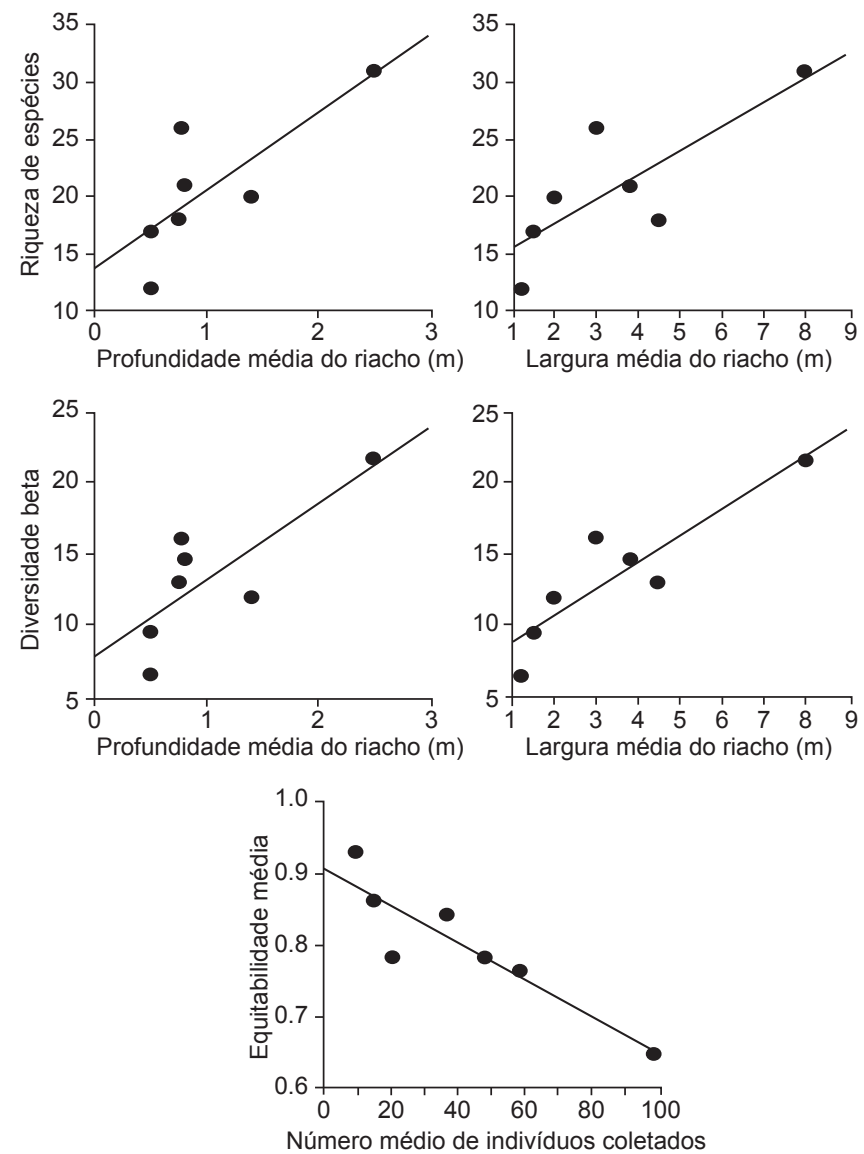

Figura 2. Diagrama de dispersão entre os descritores das comunidades de peixes (riqueza, diversidade beta e equitabilidade) e as características ambientais e número de indivíduos nos riachos amostrados na bacia do rio Ivinhema, alto rio Paraná.

Figure 2. Scatterplot of communities descriptors (richness, beta diversity and evenness) and environmental characteristics and number of individuals in the sampled streams in Ivinhema River basin, upper Paraná River. 
Tabela 3. Resultados das análises de Kruskal-Wallis e de Co-Variância para a variação espacial e temporal nos atributos das comunidades de peixes nos riachos estudados na bacia do rio Ivinhema, Alto Rio Paraná, entre Janeiro e Dezembro/2002.

Table 3. Results of the Kruskall-Wallis and co-variance analysis for the spatial and temporal variation in the fish communities attributes of sampled streams in the Ivinhema river basin, from January to December/2002.

\begin{tabular}{lccccc}
\hline \multicolumn{1}{c}{ Var. resposta } & Var. explanatória & $\mathbf{r}^{2}$ & $\mathbf{F}$ & Kruskall & Número de indivíduos \\
\hline Riqueza & Riachos & 0,501 & $4,82 * * *$ & - & $14,70 * * *$ \\
Riqueza & Meses & 0,419 & $1,20 \mathrm{~ns}$ & - & $37,42 * * *$ \\
Equitabilidade & Riachos & - & - & $38,06 * * *$ & - \\
Equitabilidade & Meses & - & - & $4,14 \mathrm{~ns}$ & - \\
\hline
\end{tabular}

ns = Não significativo; $* * *=$ Significativo a alfa 0,001 .

$\mathrm{ns}=$ Non significant; $* * *=$ Significantly to alfa 0.001 .

Tabela 4. Resultado da Análise de Correspondência Canônica (CCA) para as comunidades de peixes em riachos da bacia do rio Ivinhema, Alto Rio Paraná.

Table 4. Results of the Canonical Correspondence Analysis (CCA) for the stream fish communities in Ivinhema river basin, Upper Paraná Basin.

\begin{tabular}{lrrc}
\hline \multicolumn{1}{c}{ Descritores ambientais } & Eixo 1 & Eixo 2 & $\mathbf{r}^{\mathbf{2}}$ \\
\hline Velocidade da correnteza & $-0,98$ & $-0,22$ & $0,43 \mathrm{~ns}$ \\
Transparência da água & $-0,87$ & $-0,50$ & $0,66 \mathrm{~ns}$ \\
Profundidade do riacho & 0,99 & 0,15 & $0,60 \mathrm{~ns}$ \\
Largura do riacho & 0,93 & 0,37 & $0,82^{*}$ \\
Resumo estatístico & & & \\
Variação explicada $(\%)$ & $39 \%$ & $20,8 \%$ & - \\
\hline
\end{tabular}

ns = Não significativa; $*=$ Significativa a $\alpha=0,05$.

$\mathrm{ns}=$ Non significant; $*$ Significantly to $\alpha=0.05$.
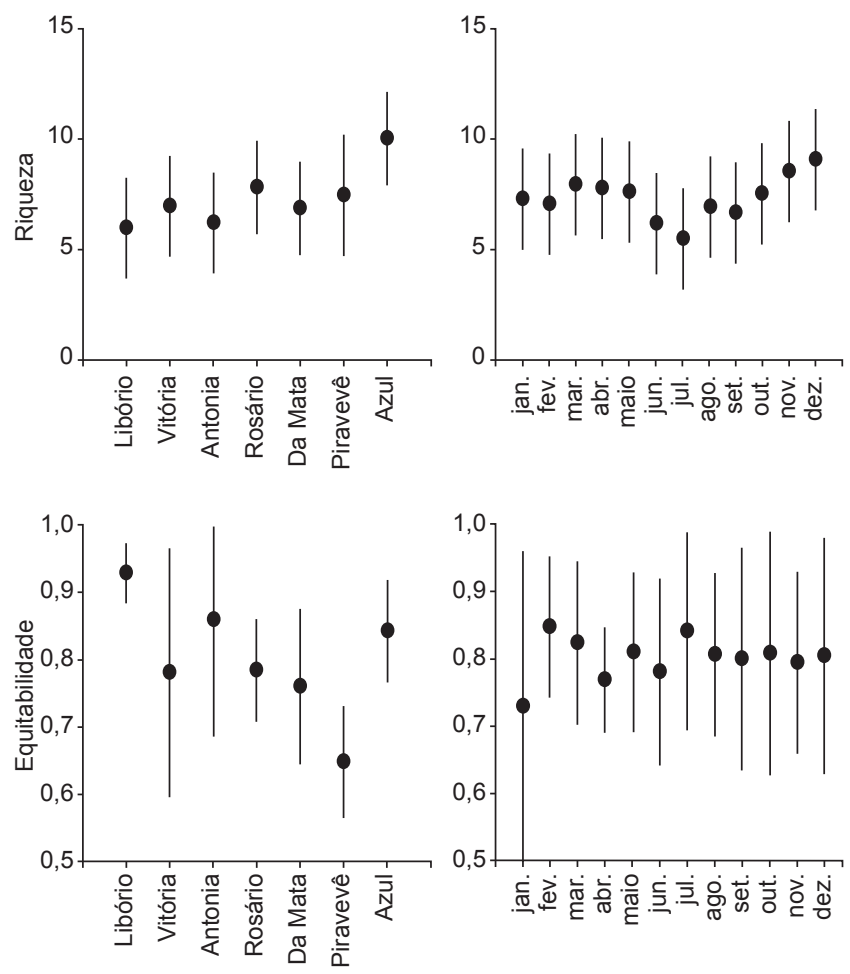

Figura 3. Variação espacial e temporal na riqueza de espécies e equitabilidade nos riachos amostrados na bacia do Rio Ivinhema, alto rio Paraná. = Média, $I=$ Intervalo de Confiança $(\alpha=0,05)$.

Figure 3. Spatial and temporal variation in species richness and evenness in sampled streams in Ivinhema river basin, upper Paraná River. = Mean, $I=$ Confidence Interval $(\alpha=0.05)$.
Coeficiente de Correlação Cofenética $r=0,77$

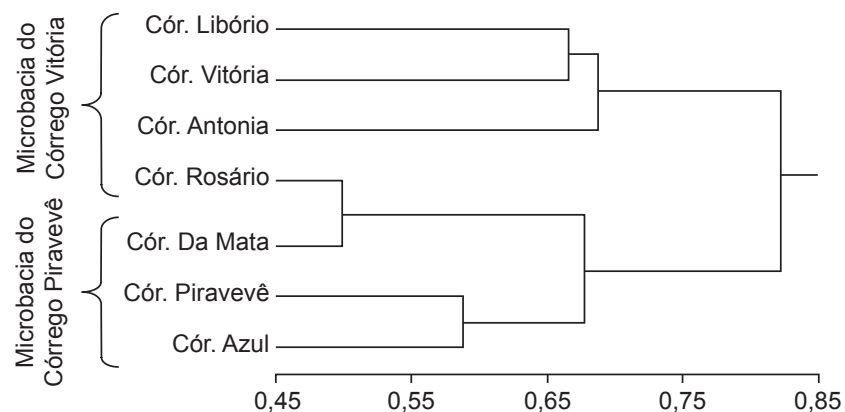

Figura 4. Dendrograma de similaridade entre os riachos amostrados na bacia do rio Ivinhema, alto rio Paraná com base na abundância total das espécies de peixes (coeficiente de Bray Curtis) coletadas entre Janeiro e Dezembro/2002.

Figure 4. Similarity dendrogram among the sampled streams in Ivinhema River basin, upper Paraná River, using the total fish species abundance (Bray Curtis coefficient) sampled from January to December/2002.

Moenkhausia sanctaefilomenae (Steindachner, 1907) e Hemigrammus marginatus foram mais abundantes em riachos mais volumosos, com pouca velocidade da correnteza e transparência da água (Figura 5), sendo que $S$. notomelas, localizando-se na região mais central do gráfico, mostrou-se pouco influenciada pelas características analisadas dos riachos.

\section{Discussão}

O número registrado de espécies (46) representa cerca de 19,5\% do número de espécies de peixes autóctones documentadas na bacia do alto rio Paraná no Brasil (Langeani et al. 2007). Esta riqueza pode ser considerada proporcionalmente elevada, se considerada a pouca representatividade destas microbacias em relação à área total da bacia do alto rio Paraná, e pode ser explicada pela variedade de habitats analisados, uma vez que os trechos coletados representavam desde riachos com elevada correnteza até ambientes com pequenas áreas de planície de inundação. Além disso, a riqueza registrada encontra-se pouco abaixo da observada por outros estudos realizados com maior número de locais amostrados, a exemplo de Castro et al. (2003, 2004,2005 ), ainda que a variação temporal (não significativa) possa ter acrescido espécies nos riachos por nós amostrados. De forma complementar, a estimativa de riqueza de espécies para os riachos amostrados mostrou-se próxima da riqueza encontrada, sugerindo que as amostragens realizadas foram eficientes para caracterizar a composição de espécies nestes riachos.

A correlação entre a riqueza de espécies e o volume dos riachos amostrados é reconhecida há muito tempo, como resultado da relação 


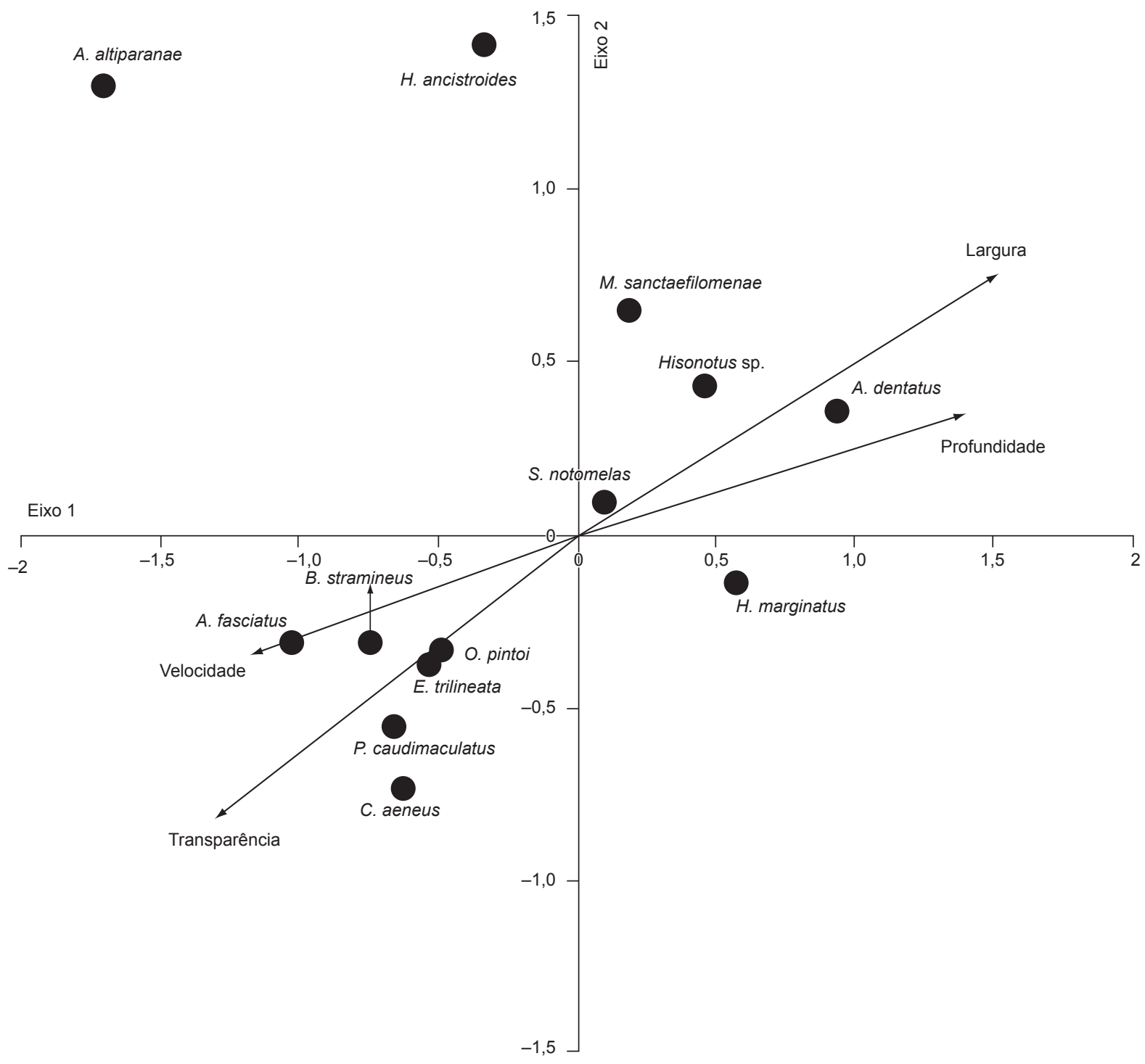

Figura 5. Diagrama de dispersão da análise de correspondência canônica (CCA) entre a abundância das principais espécies de peixes e os descritores dos riachos na bacia do rio Ivinhema, alto rio Paraná entre Janeiro e Dezembro/2002.

Figure 5. Scatterplot of Canonical Correspondence Analysis (CCA) between the main fish species and streams descriptors in Ivinhema River basin, upper Paraná River from January to December/2002.

espécie-área (MacArthur \& Wilson 1967), tendo sido demonstrada por diversos estudos (e.g., Gorman \& Karr 1978, Angermeier \& Schlosser 1989). Neste sentido, o maior volume dos riachos levaria à maior diversificação de nichos e permitiria a coexistência de maior número de espécies.

Taylor \& Warren (2001) sugerem que nos trechos inferiores das bacias hidrográficas, as comunidades de peixes são mais influenciadas pela dinâmica da migração, uma vez que as condições hidrológicas destes riachos são menos restritivas, o que é corroborado pelos resultados de Pavanelli \& Caramaschi (2003), que apontaram maior importância da migração planície/riacho no trecho final das microbacias analisadas no alto rio Paraná. Desta forma, como os locais aqui amostrados encontram-se relativamente distantes da calha do rio Ivinhema e da maior parte de sua planície de inundação, a pouca variação temporal na diversidade e composição de espécies de peixes, assim como a forte variação espacial (entre locais) constatada, sugerem maior importância das características espaciais do que temporais sobre a organização das comunidades, minimizando a importância do pulso de inundação sobre as comunidades estudadas. De forma complementar, mesmo as pequenas migrações reprodutivas das espécies residentes nestes riachos não se mostraram suficientes para alterar o padrão geral de organização das comunidades, o que também foi verificado por Grossman et al. (1998) em riachos da Carolina do Norte (EUA).

A maior taxa de migração em riachos de porções inferiores da bacia e com maior volume, constatada por Taylor \& Warren (2001), também explica a maior diversidade beta observada em riachos de maior volume, uma vez que, considerando a medida de diversidade beta adotada no presente trabalho, esta pode ser assumida como uma 
medida da taxa de turnover na composição de espécies ao longo do ano.

A formação de grupos de riachos de acordo com as microbacias amostradas sugere influência de fatores regionais (ainda que em pequena escala) sobre a composição de espécies nos riachos amostrados, assim como constatado por Matthews \& Robison (1998) para riachos em Arkansas (EUA), sendo que o córrego Rosário é o único que se isola dos demais pertencentes à mesma microbacia. Por outro lado, o resultado do teste de Mantel contradiz a diferenciação entre microbacias, sugerindo que a distância entre locais amostrados não é significativa. Esta contradição pode ser explicada pelo comportamento diferenciado do córrego Rosário (maior similaridade com a microbacia do córrego Piravevê) e pelo fato de poucos riachos terem sido amostrados (7 riachos), o que diminui o número possível de aleatorizações e aumenta a probabilidade de aceitar a hipótese nula (ausência de influência da proximidade entre riachos).

A análise de agrupamento, realizada com as abundâncias totais das espécies, demonstra que a menor dissimilaridade observada entre os riachos foi de cerca de $50 \%$, demonstrando, mais uma vez, que os riachos amostrados apresentam elevada diversidade $\beta$.

A não constatação de variação temporal nas comunidades de peixes verificada no presente trabalho, já foi constatada em outros estudos (Súarez \& Petrere Júnior 2003, Valério et al. 2007), sendo que Súarez \& Petrere Júnior (2003) constataram pouca importância da variação temporal sobre os grupos de espécies co-ocorrentes na bacia do rio Iguatemi, sugerindo que, apesar de existirem pequenas migrações nestas comunidades, estas não influenciam de forma significativa o padrão de distribuição das espécies em nível de bacia hidrográfica, desta forma as diferenças espaciais na estrutura dos habitats e características hidrológicas assumem maior importância que a variação temporal na organização das comunidades.

Além da menor diversificação de nichos em riachos de menor volume, a menor oferta de alimento, resultado da menor produtividade e as características hidrológicas limitantes podem funcionar como "filtro de espécies" (Poff 1997) permitindo que poucas espécies, do conjunto de possíveis colonizadores ocupem aqueles ambientes (Martin-Smith 1998, Valério et al. 2007, Súarez \& Petrere-Júnior 2007). Sendo assim, as diferenças estruturais entre os riachos parecem atuar como fontes primárias de organização das comunidades, atuando diretamente sobre a dinâmica da colonização e extinção das espécies de peixes (Súarez et al. 2007), sendo relativamente independentes das variações temporais, o que indica um padrão determinístico de co-ocorrência das espécies e reforça a idéia de uma forte associação entre as espécies e a estrutura dos habitats nos riachos estudados, fato também visualizado na análise de correspondência canônica.

Apesar das variáveis ambientais atuarem como filtro de espécies, contudo, diminuindo a possibilidade de uma espécie pouco adaptada às condições hidrológicas locais colonizar e persistir em locais hidrologicamente "não adequados", estes fatores não explicam toda a variação na composição e diversidade nas comunidades. O córrego Rosário, diferente dos demais na mesma microbacia apresenta-se mais similar aos córregos da Mata, Piravevê e Azul, pertencentes a outra microbacia e com características hidrológicas diferentes. Este trecho amostrado apresenta grande concentração de gramíneas flutuantes (Poaceae), bem como Typha sp. em um pequeno trecho alagável, alternando-se com trechos de maior correnteza (à montante $\mathrm{e}$ jusante), desta forma, é possível que em anos com menor pluviosidade e consequentemente velocidade da correnteza, indivíduos das espécies de peixes características de ambientes de planície de inundação tenham colonizado este local e encontrando condições adequadas à sua sobrevivência tornando-se abundantes, a despeito das diferenças entre este local e os demais da mesma microbacia.
Assim, as características locais (estrutura do habitat) atuariam em conjunto com características regionais (separação entre microbacias) e históricas (dinâmica de colonização e extinção pontual) na determinação das assembléias de peixes conduzindo locais com diferenças ambientais a possuírem conjuntos diferentes de espécies, mesmo oriundas de um mesmo conjunto de potenciais colonizadores, sendo esta dinâmica pouco influenciada pelas variações hidrológicas temporais nos locais amostrados.

\section{Agradecimentos}

A Suely A. D. Molina, Amal Farhat, Priscilla Gobi, Alexandro C. Florentino e Luzia S. Lourenço pelo auxílio nos trabalhos de campo. A Flávio C. T. Lima (Museu de Zoologia da Universidade de São Paulo), pelo auxílio na identificação das espécies. À UEMS pelo apoio financeiro, à Lilian Casatti e aos avaliadores que contribuíram muito para a melhoria do manuscrito.

\section{Referências Bibliográficas}

ABES, S.S. \& AGOSTINHO, A.A. 2001. Spatial patterns in fish distributions and structure of the ichthyocenosis in the Água Nanci stream, upper Paraná river basin, Brazil. Hydrobiol. 445(1-3):217-227.

AGOSTINHO, A.A. \& PENCZACK, T. 1995. Populations and production of fish in two small tributaries of the Paraná River, Paraná, Brazil. Hydrobiol. 312(3):153-166.

ANGERMEIER, P.L. \& KARR, J.R. 1983. Fish communities along environmental gradients in a system of tropical streams. Environ. Biol. Fishes 9(2):117-135.

ANGERMEIER, P.L. \& SCHLOSSER, I.J. 1989. Species-area relationships for stream fishes. Ecology 70(5):1450-1462.

BRITSKI, H.A., SILIMON, K.Z. \& LOPES B.S. 1999. Peixes do Pantanal, Manual de identificação. Embrapa-SPI, Brasília; Embrapa-CPAP, Corumbá.

BRITSKI, H.A. \& LANGEANI, F. 1988. Pimelodus paranaensis, sp. n., um novo Pimelodidae (Pisces, Siluriformes) do Alto Paraná, Brasil. Rev. Bras. Zool. 5(3):409-417.

CASATTI, L. 2004. Ichthyofauna of two streams (silted and reference) in the Upper Paraná River basin, southeastern Brazil. Braz. J. Biol 64(4):757-765.

CASATTI, L., LANGEANI, F., SILVA, A.M. \& CASTRO, R.M.C. 2006. Stream fish, water and habitat quality in a pasture dominated basin, Southeastern Brazil. Braz. J. Biol. = Rev. Bras. Biol. 66(2b):681-696.

CASTRO, R.M.C., CASATTI, L., SANTOS, H.F., FERREIRA, K.M., RIBEIRO, A.C., BENINE, R.C., DARDIS, G.Z.P., MELO, A.L.A., ABREU, T.X., BOCKMANN, F.A., CARVALHO, M., GIBRAN, F.Z. \& LIMA, F.C.T. 2003. Estrutura e composição da ictiofauna de riachos do Rio Paranapanema, sudeste e sul do Brasil. Biota Neotrop. 3(1): http:// www.biotaneotropica.org.br/v3n1/pt/abstract?article+BN01703012003 (último acesso em 12/12/2007)

CASTRO, R.M.C., CASATTI, L., SANTOS, H.F., MELO, A.L.A., MARTINS, L.S.F., FERREIRA, K.M., GIBRAN, F.Z., BENINE, R.C., CARVALHO, M., RIBEIRO, A.C., ABREU, T.X., BOCKMANN, F.A., DARDIS, G.Z.P., STOPIGLIA, R. \& LANGEANI, F. 2004. Estrutura e composição da ictiofauna de riachos da bacia do Rio Grande, no Estado de São Paulo, Sudeste do Brasil. Biota Neotrop. 4(1): http://www.biotaneotropica. org.br/v4n1/pt/abstract?article+BN0170402004 (último acesso em 13/12/2007)

CASTRO, R.M.C., CASATTI, L., SANTOS, H.F., VARI, R.P., MELO, A.L.A., MARTINS, L.S.F., ABREU, T.X., BENINE, R.C., GIBRAN, F.Z., RIBEIRO, A.C., BOCKMANN, F.A., CARVALHO, M., PELIÇÃO, G.Z., FERREIRA, K.M., STOPIGLIA, R. \& AKAMA, A. 2005. Structure and composition of the stream ichthyofauna of four tributary rivers of the upper Rio Paraná basin, Brazil. Ichthyol. Explor. Freshwaters 16(3):193-214 
CASTRO, R.M.C. \& CASATTI, L. 1997. The fish fauna from a small forest stream of the upper Paraná River Basin, southeastern Brazil. Ichthyol. Explor. Freshwaters 7(4):337-352.

CETRA, M. \& PETRERE-JÚNIOR, M. 2006. Fish assemblage structure of the Corumbataí River Basin, São Paulo State, Brazil: characterization and anthropogenic disturbances. Braz. J. Biol. = Rev. Bras. Biol. 66(2A):431-439.

CLARKE, K.R. 1993. Non-parametric multivariate analysis of changes in community structure. Aust. J. Ecol. 18(1):117-143.

FERREIRA, C.P. \& CASATTI, L. 2006. Influência da estrutura do habitat sobre a ictiofauna de um riacho em uma micro-bacia de pastagem, São Paulo, Brasil. Rev. Bras. Zool. 23(3):642-651.

GORMAN, O.T. \& KARR, J.R. 1978. Habitat structure and stream fish communities. Ecology 59(3):507-515.

GRAÇA, W.J. \& PAVANELLI, C.S. 2007. Peixes da planície de inundação do alto rio Paraná e áreas adjacentes. EDUEM, Maringá. 241p.

GROSSMAN, G.D., RATAJCZAK, R.E., CRAWFORD, M. \& FREEMAN, M.C. 1998. Assemblage organization in stream fishes: effects of environmental variation and interspecific interactions. Ecol. Monogr. 68(3):395-420

HEINO, J., MYKRÄ, H. \& MUOTKA, T. 2007. Ecological filters and variability in stream macroinvertebrate communities: do taxonomic and functional structure follow the same path? Ecography 30(2):217-230.

LANGEANI, F., CASTRO, R.M.C., OYAKAWA, O.T., SHIBATTA, O.A., PAVANELLI, C.S \& CASATTI, L. 2007. Diversidade da ictiofauna do alto rio Paraná: composição atual e perspectivas futuras. Biota Neotrop. 7(3): http://www.biotaneotropica.org.br/v7n3/pt/ abstract?article+bn03407032007 (último acesso em 13/12/2007)

MACARTHUR, R.H. \& WILSON, E.O. 1967. The theory of island biogeography. Princeton University Press, Princeton.

MACARTHUR, R., RECHER, H. \& CODY, M. 1966. On the relation between habitat selection and species diversity. Am. Nat. 100(913):319-332.

MARTIN-SMITH, K.M. 1998. Relationships between fishes and habitat in rainforest streams in Sabah, Malaysia. J. Fish Biol. 52(3):458-482.

MATTHEWS, W.J. \& ROBISON, H.W. 1998. Influence of drainage connectivity, drainage area and regional species richness of fishes of the interior highlands in Arkansas. Am. Midl. Nat. 139(1):1-19.

OBERDORFF, T., PONT, D., HUGUENY, B. \& CHESSEL, D. 2001. A probabilistic model characterizing fish assemblages of French rivers: a framework for environmental assessment. Freshwater Biol. 46(3):399-415.

PAVANELLI, C.S. \& CARAMASCHI, E.P. 2003. Temporal and spatial distribution of the ichthyofauna in two streams of the upper Rio Paraná Basin. Braz. Arch. Biol. Technol. 46(2):271-280.

PENCZACK, T., AGOSTINHO, A.A. \& OKADA, E.K. 1994. Fish diversity and community structure in two small tributaries of the Paraná river, Paraná State, Brazil. Hydrobiol. 294(3):43-251.
POFF, N.L. 1997. Landscape filters and species traits: towards mechanistic understanding and prediction in stream ecology. J. N. Am. Benthol. Soc. 16(2):391-409.

R DEVELOPMENT CORE TEAM. 2005. R: A language and environment for statistical computing. [2.2.0]. R Foundation for Statistical Computing, Vienna.

RAHEL, F.J. \& STEIN, R.A. 1988. Complex predator-prey interactions and predator intimidation among crayfish, piscivorous fish, and small benthic fish. Oecologia 75(1):94-98.

SÚAREZ, Y.R. 2008. Fish, lower Ivinhema river basin streams, state of Mato Grosso do Sul, Brazil. Check List 4(3):226-231.

SÚAREZ, Y.R. \& PETRERE-JÚNIOR, M. 2007. Environmental factors predicting fish communities structure of two neotropical rivers in Brazil. Neotrop. Ichthyol. 5(1):61-68.

SÚAREZ, Y.R., VALÉRIO, S.B., TONDATO, K.K., XIMENES, L.Q.L. \& FELIPE, T.R.A. 2007. Determinantes ambientais da ocorrência de espécies de peixes em riachos de cabeceira da bacia do rio Ivinhema, alto rio Paraná. Acta Sci., Biol. Sci. 19(2):145-150.

SÚAREZ, Y.R. \& PETRERE-JÚNIOR, M. 2003. Associações de espécies de peixes em ambientes lóticos da bacia do rio Iguatemi, Estado do Mato Grosso do Sul. Acta Sci., Biol. Sci. 25(2):361-367.

SÚAREZ, Y.R. \& PETRERE-JÚNIOR, M. 2005. Organização das assembléias de peixes em riachos da bacia do rio Iguatemi, Mato Grosso do Sul. Acta Sci., Biol. Sci. 22(2):161-167.

TAYLOR, C.M. \& WARREN Jr, M.L. 2001. Dynamics in species composition of stream fish assemblages: environmental variability and nested subsets. Ecology 82(8):2320-2330.

VALÉRIO, S.B., SÚAREZ, Y.R., FELIPE, T.R.A., TONDATO, K.K. \& XIMENES, L.Q.L. 2007. Organization patterns of headwater-stream fish communities in the Upper Paraguay-Paraná basins. Hydrobiol. 583(1):241-250

WEITZMAN, S.H., Menezes, N.A. \& Weitzman, M.J. 1988. Phylogenetic biogeography of the Glandulocaudinae (Teleostei: Characiformes, Characidae) with comments on distribution of the other freshwater fishes in eastern and southeastern Brazil. In Proceedings of a workshop on neotropical distribution patterns. (P.E. Vanzolini \& W.R. Heyer, coord.). Academia Brasileira de Ciências, Rio de Janeiro. p.379-427.

WRIGHT, J.P. \& FLECKER, A.S. 2004. Deforesting the riverscape: the effects of wood on fish diversity in a Venezuelan piedmont stream. Biol. Conserv. 120(3):439-447.

ZARET, T.M. \& RAND, A.S. 1971. Competition in tropical stream fishes: support for the competitive exclusion principle. Ecology 52(2):336-342.

Recebido em 16/03/08 Versão reformulada recebida em 29/07/08 Publicado em 29/08/08 